\title{
Archives of Clinical and Biomedical Research
}

Volume 1, Issue 2

Case Report

\section{Case Report on Ruptured Renal Artery Aneurysm}

\author{
Mohammed Darouichi $^{1^{*}}$, S.Qanadli ${ }^{2}$, P.E.Constanthin ${ }^{3}$ \\ ${ }^{1}$ Department of Radiology, Champel Medical Institute, Geneva, Switzerland \\ ${ }^{2}$ Department of Radiology, Lausanne, CHUV, Switzerland \\ ${ }^{3}$ Department of Fundamental Neurosciences, Genève, CMU, Switzerland \\ *Corresponding Author: Mohammed Darouichi, Department of Radiology, Champel Medical Institute, Geneva, \\ Switzerland, E-mail: Mohammed.Darouichi@ne.ch
}

Received: 10 April 2017; Accepted: 24 April 2017; Published: 27 April 2017

\begin{abstract}
Renal aneurysms are a rare entity with an incidence of $0.09 \%$ of the population. They are often asymptomatic and frequently discovered incidentally during abdominal imaging for unrelated causes. Rupture of renal artery aneurysms is a surgical emergency, very often lethal. Swift diagnosis is crucial and is made by ultrasound and abdominal MDCT. We report the case of a renal artery rupture in a 58 year-old man who arrived in the emergency department with left flank pain of sudden onset followed by a hypovolemic shock. MDCT scan showed the rupture of an aneurysm of the left renal artery associated with a retroperitoneal hematoma. It is a life-threatening situation in case of delayed diagnosis. In our case, prompt imaging and diagnosis led to conservative treatment and nephrectomy was avoided.
\end{abstract}

Keywords: Renal Aneurysms; Nephrectomy; Aneurysmectomy

\section{Introduction}

Renal artery aneurysms are a rare entity and their complications such as spontaneous rupture are uncommon. Rupture of renal artery aneurysm is considered to be a surgical emergency that presents with sudden onset of flank pain, hematuria and hypovolemic shock due to retroperitoneal haemorrhage. Swift and accurate diagnosis of this rare entity allows immediate indications for treatment, which include endovascular approach or surgical treatment. 


\section{Case Report}

A 58-year-old man with no prior medical history presented to the emergency department with severe left flank pain of sudden onset. While being treated in the emergency department he developed a hypovolemic shock. On physical examination the patient presented with a mild sensibility of the left flank without any other gastrointestinal or urogenital symptoms. Blood analysis results revealed hemoglobin at $14.2 \mathrm{~g} / \mathrm{dl}$ (14-18), creatinine at $1.6 \mathrm{mg} / \mathrm{dl}$ (0.61.2),and urea at $51 \mathrm{mg} / \mathrm{dl}(15-50)$.

An abdominal CT scan was performed without and after intravenous injection of contrast medium, with $1.5 \mathrm{~mm}$ slice width and axial and coronal reconstructions. CT images in the arterial phase demonstrated an aneurysm of the left renal artery (diameter: $2.8 \mathrm{~cm}$ ) as well as an intermittent bleeding in the retroperitoneal space. The hematoma occupied the entire left anterior renal space measuring $20 \mathrm{~cm}$ in length. The source of this retroperitoneal haemorrhage was the left renal artery aneurysm rupture and more precisely its anterior-superior part, leading to an active bleeding (Fig A, B, C and D).

Delayed CT images showed a discrete delay in parenchymal secretory phase in the superior pole of the left kidney in comparison to the right kidney. No renal or ureteral lesion was detected. The patient was immediately transferred to two weeks.

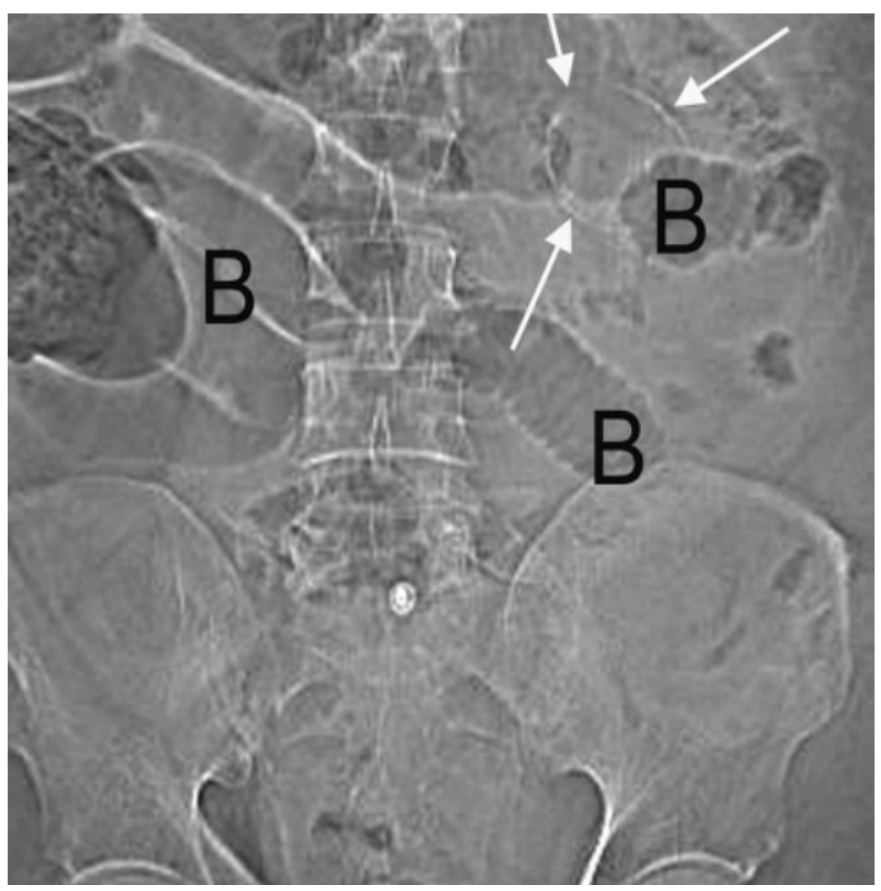

Figure A: Plain abdominal X-ray may demonstrate calcifications (see arrows) of the aneurysm, or an intestinal ileus due to irritation of the retro peritoneum from the hematoma. 


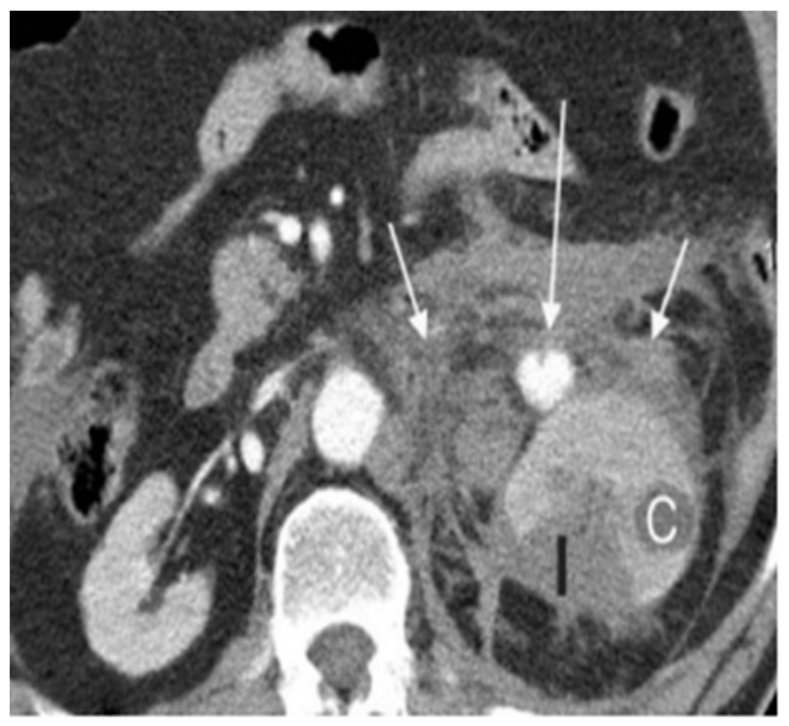

Figure B: Ruptured aneurysm. The aneurysm retains contrast material while the adjacent vessels are washed out.
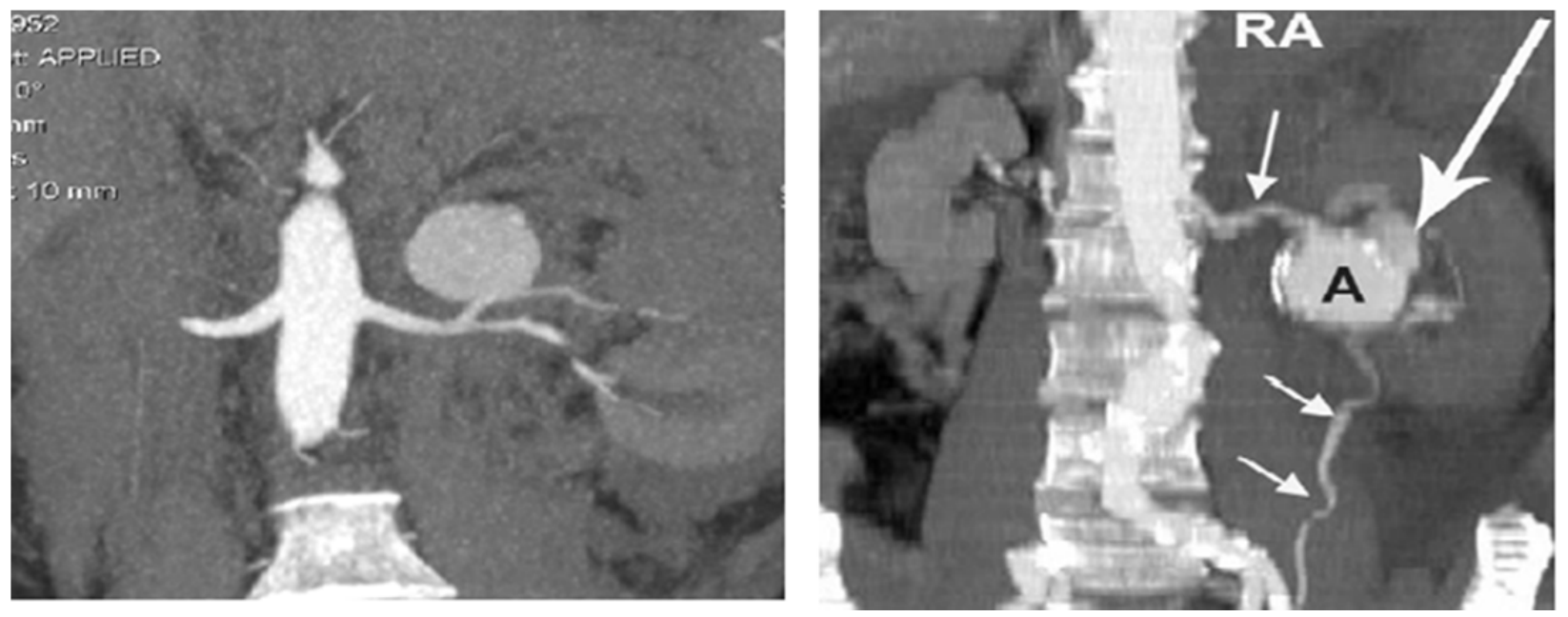

Figure C, D: Coronal MIP shows 2 x $2 \mathrm{~cm}$ aneurysm ( A, wide large arrow) of the distal portion of left renal artery.

\section{Discussion}

Renal artery aneurysms are uncommon with an estimated incidence of $0.015-0.09 \%$ in the general population. They account for $25 \%$ of al visceral aneurysms and occur more often in the female population, generally at the $4^{\text {th }}-6^{\text {th }}$ decade [1]. They are primarily located on the right artery and are unilateral in $80 \%$ of the cases, bilateral in $10-25 \%$ and multiple with a range of 1-30\% [2]. 
Poutasse classification established in 1975 demonstrates 4 structural types of aneurysms of the renal arteries [3]:

1) Saccular aneurysm: the most common type, representing $80 \%$ of all renal artery aneurysms. It is frequently located at the bifurcation of the renal artery.

2) Fusiform aneurysm, located at the bifurcation or branches of the renal artery, often in association with fibromuscular dysplasia.

3) Dissecting aneurysm, often located in the branches of renal artery.

4) Intrarenal micro aneurysms.

Pathophysiology of renal aneurysms formation is not yet certain, but their development is related to atherosclerosis and fibro-muscular dysplasia in $60 \%$ of the cases, and to renal arterial hypertension in $25 \%$ of the case [4]. Lumsden reported a prevalence of 39\% of renal artery aneurysms in patients with hypertension that were unresponsive to medical treatment [5]. However, several cases, like our patient, occur in patients with no previous medical history and those cases are particularly difficult to identify due to potential lack of suspicion from physicians.

Peter $\mathrm{K}$ et al. reported that the histological finding in 56 aneurysms was important atherosclerotic changes in 9 cases, dysplastic modifications in 26 and other non-specific fibrotic changes in 21 cases [6]. This study was made over a period of 35 years of experience with 252 diagnosed aneurysms of the renal artery of which 168 were surgically treated [7].

Renal artery aneurysms are frequently asymptomatic and in the majority of the cases are discovered incidentally during abdominal imaging. However, in the case in which a complication occurs, physicians need to be aware of them and to act quickly for their consequences can be dire very quickly (In our case, the patient developed hypovolemic shock two hours after admission). Frequent complications of aneurysms of the renal arteries include dissection, arterial thrombosis, renal infarction and/or spontaneous rupture with haemorrhage [8].

Spontaneous rupture of the aneurysm is the most life-treating situation with a high rate of mortality, up to 80\% [9]. Predisposing factors for rupture are pregnancy, intrarenal location of the aneurysms, partial calcified aneurysms, hypertension and an aneurismal diameter bigger than $2 \mathrm{~cm}$ [10]. For pregnancy, high risk is established at the $3^{\text {rd }}$ trimester [11]. The evolution towards the rupture of a renal aneurysm is sometimes spontaneous, after a traumatism or due to certain diseases like LEN, Behcet’s disease, Marfan’s syndrome or fibro-muscular dysplasia [12].

When patients suffer from a ruptured renal artery aneurysm, they typically present with flank pain of sudden onset complicated by a hypovolemic shock due to bleeding in the retro-peritoneum [13].

In our case, our patient presented with left flank pain and signs of hypovolemic shock that manifested approximately 2 hours after admission in the emergency department. The ultrasound and CT scan promptly demonstrated the rupture of the renal artery aneurysm and its complications. 
Discovered incidentally or due to clinical suspicion in the presence of a complication, diagnosis of renal artery aneurism is purely radiologic and comprises the following technics.

Plain abdominal X-ray may demonstrate calcifications of the aneurysm or an intestinal ileus due to irritation of the retro-peritoneum from the hematoma.

Doppler ultrasound, can demonstrate the renal artery aneurysm, its localization, dimensions and characteristics. Contrast-enhanced CT confirms the aneurysm and defines anatomic details as well as demonstrating extravasations of contrast in case of active bleeding and quantifies the retroperitoneal hematoma [14]. Angiography is the imaging modality of choice. Currently it is reserved for the cases of embolization or stenting of renal aneurysms [15].

Despite the recent developments of endovascular treatment of complicated renal artery aneurysms [16, 17], surgery remains a very important treatment in case of a ruptured renal aneurysm (determined by the classification of the aneurysm), particularly in the presence of hypovolemic shock or renal vascular malformation [18]. Indications for surgical treatment include rupture of renal aneurysm in pregnant patients, renal ischemia, dissecting aneurysms, aneurysm in a unique kidney, or an aneurysm diameter superior to $2 \mathrm{~cm}$ [19]. Nephrectomy or partial nephrectomy is reserved for patients with renal infarct or severe renal atrophic ischemia. Aneurysmectomy is indicated more for saccular aneurysms or after auto-transplantation [20]. Auto-transplantation is recommended for intrarenal extending aneurysms.

\section{Conclusion}

Even though renal aneurysms are a rare entity, diagnosis is made more frequently due to abdominal imaging. Rupture of renal artery aneurysms with following haemorrhage into the retro-peritoneum is a life threatening circumstance, as proven again with this case in which hypovolemic shock developed only two hours after patient

admission. Accurate diagnosis with the aid of MDCT contrast enhanced exams as well as angiography permits swift management of the patient with either immediate surgery or endovascular treatment.

\section{References}

1. Edwards BS, Stanson AW, Holley KE, Sheps SG. Isolated spontaneous renal artery dissection presentation, evaluation, management and pathology. Mayo Clin Proc 57 (1982): 564-571.

2. Tham G, Ekelund L, Herrlin K. Renal artery aneurysms. Natural history and prognosis Ann Surg 197 (1983): 348

3. Poutasse EF. Renal artery aneurysms. The Journal of Urology 113 (1975): 443-449.

4. Lumsden AB, Salam TA, Walton KG. Renal artery aneurysm: a report of 28 cases. Cardiovasc Surg Apr 4 (1996): 185-189

5. Romero-Terán O, Torres-Contreras et al. Calcified renal artery aneurism and high blood pressure. A case report and review of the literature. Cir Cir 72 (2004): 217-220. 
6. Lumsden AB, Salam TA, Walton KG. Renal artery aneurysm: a report of 28 cases. Cardiovasc Surg 4 (1996): 185-189.

7. Henke PK, Cardneau JD, Welling Iii TH, Upchurch Jr GR, Wakefield TW, et al. Renal artery aneurysms: a 35year clinical experience with 252 aneurysms in 168 patients. Annals of surgery 234 (2001): 454-463.

8. Stanley JC, Rhodes EL, Gewertz BL, et al. Renal artery aneurysms. Significance of macroaneruysms exclusive of dissections and fibrodysplastic mural dilations. Arch Surg 110 (1975): 1327-1333.

9. Henricksson C, Bjorkerud S, Nilson A. Natural history of renal artery aneurysm elucidated by repeated angiography and pathoanatomical studies. Eur Urol 11 (1985): 244-248.

10. Mercier C, Piquet P, Piligian F, et al. Aneurysms of the renal artery and its branches. Ann Vasc Surg 1 (1986): 321-327.

11. Cohen JR, Shamash FS. Ruptured renal artery aneurysm during pregnancy. J Vasc Surg 6 (1987): 51-59.

12. Han K, Siegel R, Pantuck AJ, Gazi MA. Behcet's syndrome with left ventricular aneurysm and ruptured renal artery pseudoaneurysm. Urology 54 (1999): 162.

13. Henriksson C, Bjorkerud, et al. Natural history of renal artery aneurysm elucidated by repeated angiography and pathoanatomical studies. Eur Urol 11 (1985): 244-248.

14. Zubarev AV. Ultrasound of renal wessels. Eur Radiol 11 (2001): 1902-1915.

15. Perona F, Barile A, Belli AM, Ferro C. Ruptured suprarenal artery aneurysm: angiographic diagnosis and treatment by selective embolization. Cardiovasc Intervent Radiol 19 (1996): 291-293.

16. Nassiri N, Huntress LA. Stent-Assisted Coil Embolization of a Symptomatic Renal Artery Aneurysm at a Bifurcation Point. Ann Vasc Surg 2017 in Press.

17. Siani A, Gabrielli R, Accrocca F, Marcucci G. Successful covered stent-graft repair in symptomatic renal artery aneurysm early after childbirth. J Cardiovasc Surg 58 (2017): 95-98.

18. Franz RW, Tanga CF. Treatment of Complex, Combined Renal Artery Aneurysm and Renal Arteriovenous Fistula with Nephrectomy. Int J Angiol 26 (2017): 068-072.

19. Dzsinich C, Gloviczki P, McKusick MA, et al. Surgical management of renal artery aneurysm. Cardiovasc Surg 1 (1993): 243-247.

20. Hupp T, Allenberg JR, Post K, et al. Renal artery aneurysm: surgical indications and results. Eur J Surg 6 (1992): 477-486.

This article is an open access article distributed under the terms and conditions of the Creative Commons Attribution (CC-BY) license 4.0 